beam diffraction is used to characterise the substrate which is a LiF single crystal. Monochromatic diffraction is used to analyse the thin film, the grain constituting the film having a nanometric size. With the large two dimensional detector available at the beamline, partial powder rings are collected. The thin film diffraction patterns are analysed using the classical powder diffraction technique and $\sin ^{2}$ method.

[1] A.A.MacDowell, R.S.Celestre, N.Tamura, R.Spolenak, B.C. Valek, W.L.Brown, J.C.Bravman, H.A.Padmore, B.W.Batterman \& J.R.Patel, Nuclear Instruments and Methods in Physics Research A 2001, 936-943, 467

\section{MS28 O4}

Recent developments in single crystal microdiffraction at the ESRF-ID13 beamline. D. Popov ${ }^{*}, M$. Burghammer and C. Riekel ESRF, B.P.220, F-38043 Grenoble Cedex09, France. E-mail: popov@esrf.fr

\section{Keywords: microdiffraction, microgoniometer, microcrystals}

The aim of this contribution is to report on the state of microcrystallography at the ESRF-ID13 undulator beamline. Single crystal experiments on a wide range of samples are usually performed using a microgoniometer with beam sizes down to 5 m.[1] A new microgoniometer has been developed for single crystal diffraction with a $1 * 1 \quad \mathrm{~m}^{2}$ beam.[2] The sample preparation is based on Kleindiek Nanotechnik "nanomanipulator", which is installed at an Olympus BX51W1 microscope. Small unit-cell microcrystals are usually glued to glass capillaries and mounted on the microgoniometer. Protein samples are usually cryofrozen in loops. The beam is focused by parabolic compound $\mathrm{Be}$ refractive lenses [3] and collimated to 5,10 or $30 \mu \mathrm{m}$. The $1^{*} 1 \mathrm{~m}^{2}$ beam system is based on KB mirrors or Fresnel lenses focusing.[2] Mechanical stability is obtained by an air-bearing rotation stage with sub micron sphere of confusion. Centering of the sample on the goniometer is done semiautomatically by a Kleindiek Nanotechnik manipulater mounted on the rotation stage. This contribution will show several case studies performed with the microgoniometer (A-amylose) and the $1 * 1 \mathrm{~m}^{2}$ beam system (Xylanase II, a new metal organic framework compound).

[1] Riekel, C., Burghammer, M. \& Schertler, G. Curr. Opin. Struct. Biol. 15, 556-562 (2005).

[2] Moukhametzianov, R. , Burghammer, M., Edwards, P., Petitdemange, P., Fransen, M., Riekel , C., Popov, D. Protein crystallography with a micron-sized synchrotron radiation beam. To be published.

[3] Schroer, C. G.; Kuhlmann, M.; Lengeler, B.; Günzler, T. F.; Kurapova, O.; Benner, B.; Rau, C.; Simionovici, A. S.; Snigirev, A.; Snigireva, I.; Mancini, D. C., Ed.; SPIE, 2002; Vol. 4783, pp 10-18.

\section{MS28 O5}

Selective 3D imaging of diamond/C60 growth's pellet by X-Ray Micro-Diffraction Computed-Tomography Pierre Bleuet ${ }^{\mathrm{a}}$, Eleonore Welcomme ${ }^{\mathrm{b}}$, Eric Dooryhee, Philippe Walter ${ }^{\mathrm{b}}$, Jean-Louis Hodeau ${ }^{\mathrm{c}}$. ${ }^{\mathrm{a}} I D 22$, ESRF, Grenoble France. ${ }^{\mathrm{b} C e n t r e}$ de Recherche et de
Restauration des Musées de France, C2RMF, Paris, France. 'Institut Neel-CNRS, Grenoble, France. e-mail: hodeau@grenoble.cnrs.fr

Keywords: microdiffraction, tomography, C60, diamond

In this presentation, we present the mapping of nanocrystalline heterogeneous materials by using X-ray diffraction computed tomography (XRD-CT) with high spatial resolution.

Computed tomography is usually based on the detection during the moving $(\mathrm{x}, \mathrm{z})$ and the rotation of the sample of a signal such as X-ray absorption, phase contrast, Compton diffusion or X-ray fluorescence. From this data, a spatial distribution reconstruction can be performed to visualize the electron density, the mass density distribution or the distribution of all chemical elements. We have adapted the reconstruction algorithm to perform such an analysis by using the $\mathrm{X}$-ray diffraction signal. Diffraction profiles are collected in parallel projections from the sample with a 2D detector. After integration of the images, selective Bragg peaks or amorphous signals are used for the reconstruction of the different phases existing in heterogeneous samples. For this development, we used a method already developed to combine the information obtained by different types of tomography (integrated tomographic technique ITT) [1].

We demonstrate the high possibilities of X-ray diffraction computed tomography (XRD-CT) by mapping different nano-crystallized diamond-like phases of a heterogeneous pellet synthesized from C60 molecules under High Pressure. On such a transformation, if compressed non hydrostatically at room temperature, the fullerene cages collapse, producing polycrystalline cubic diamond and an $s p^{3}$ amorphous carbon phases [2]. Using a $2 \mu \mathrm{m} X$-ray beam, the XRD-CT analysis discriminates the different phases and maps their distribution in the pellet formed in the diamond HP cell. The reliability of XRD-CT is excellent, as shown by the similar reconstruction built from the different reflections of cubic diamond or $\mathrm{sp}^{3}$ amorphous phase. Furthermore, by selecting intense pixels in the corresponding sinograms, it is possible to extract the scattering pattern of each phase even for the minor ones (less than 1\%).

Using XRD-CT analysis, we have demonstrated that even in presence of amorphous phases and reflection overlaps, it is possible to discriminate, to map and also to characterize different $s p^{3}$ carbon phases with similar density. We will also illustrate the efficiency of this method on other heterogeneous samples like mixed powders or archaeological ones.

[1] Golosio B., Simionovici A., Somogyi A., Lemelle L. Chukalina M., Brunetti A. "Internal elemental microanalysis combining X-ray fluorescence, Compton and transmission tomography", J. Appl. Phys. 2003, 94(1) 145

[2] Hodeau J.L. et al., "High Pressure Transformations of C60 to Diamond and $\mathrm{sp}^{3}$ Phases at room temperature and to $\mathrm{sp}^{2}$ Phases at high temperature". Phys. Rev., 1994, B50, 10311; Marques L. et al., "Ordering mechanism in HP polymerization of C60: Avoiding geometrical frustration by stress-driven bond selection", Phys. Rev., 2003, B68, 193408 